\title{
Methylmalonic acidemia with homocystinuria, type cblD
}

INSERM

\section{Source}

INSERM. (1999). Orphanet: an online rare disease and orphan drug data base.

Methylmalonic acidemia with homocystinuria, type cblD. ORPHA:79283

cblD type methylmalonic acidemia with homocystinuria is a form of methylmalonic acidemia with homocystinuria (see this term), an inborn error of vitamin B12 (cobalamin)

metabolism characterized by variable biochemical, neurological and hematological manifestations. 